\title{
Molecular Mechanisms Controlling Midline Crossing by Precerebellar Neurons
}

\author{
Thomas Di Meglio, ${ }^{1,2}$ Kim T. Nguyen-Ba-Charvet, ${ }^{1,2}$ Marc Tessier-Lavigne, ${ }^{3}$ Constantino Sotelo,,${ }^{1,2,4}$ and \\ Alain Chédotal ${ }^{1,2}$ \\ ${ }^{1}$ Centre National de la Recherche Scientifique, Unité Mixte de Recherche (UMR) 7102, 2Université Pierre et Marie Curie, UMR 7102, F-75005 Paris, France, \\ ${ }^{3}$ Genentech, South San Francisco, California 94080, and ${ }^{4}$ Cátedra de Neurobiología del Desarrollo "Remedios Caro Almela," Instituto de Neurociencias de \\ Alicante, Universidad Miguel Hernández de Elche-Consejo Superior de Investigaciones Científicas, 03550 San Juan de Alicante, Alicante, Spain
}

Precerebellar neurons of the inferior olive (I0) and lateral reticular nucleus (LRN) migrate tangentially from the rhombic lip toward the floor plate following parallel pathways. This process is thought to involve netrin-1 attraction. However, whereas the cell bodies of LRN neurons cross the midline, IO neurons are unable to do so. In many systems and species, axon guidance and cell migration at the midline are controlled by Slits and their receptor Robos. We showed previously that precerebellar axons and neurons do not cross the midline in the absence of the Robo3 receptor. To determine whether this signaling by Slits and the two other Robo receptors, Robo1 and Robo2, also regulates precerebellar neuron behavior at the floor plate, we studied the phenotype of Slit1/2 and Robo1/2/3 compound mutants. Our results showed that many IO neurons can cross the midline in absence of Slit $1 / 2$ or Robo1/2, supporting a role for midline repellents in guiding precerebellar neurons. We also show that these molecules control the development of the lamellation of the inferior olivary complex. Last, the analysis of Robo 1/2/3 triple mutants suggests that Robo3 inhibits Robo1/2 repulsion in precrossing LRN axons but not in $\mathrm{IO}$ axons in which it has a dominant and distinct function.

Key words: cerebellum; inferior olive; floor plate; Roundabout; Slit; migration

\section{Introduction}

At all levels of the nervous system, axons cross the midline, dorsally or ventrally, to form commissural projections (Williams et al., 2004; Lindwall et al., 2007). In most cases, only the axons project to the controlateral side, but sometimes cell bodies also cross, which ultimately leads to an ipsilateral projection (George et al., 2007) (see also below). More rarely, commissures are formed by a migration of neuronal cell bodies or the extension of dendrites across the midline (Simon and Lumsden, 1993; Furrer et al., 2003; Suli et al., 2006). In humans, defects in the formation of commissures during development often results in major brain or motor dysfunction (Engle, 2007; Paul et al., 2007).

Midline crossing has been described in virtually all animal species and the molecular mechanisms regulating this process are known to be highly conserved in evolution (Goodman, 1994). Two ligand/receptor couples play a major role in this process:

Received Jan. 8, 2008; revised April 18, 2008; accepted May 7, 2008.

This work was supported by grants from the Association pour la Recherche sur le Cancer and the Fondation pour la Recherche Médicale (Programme Équipe FRM) (A.C.). T.D.M. was supported by a fellowship from the Ministère de la Recherche et de la Technologie. M.T.-L. was supported by the Howard Hughes Medical Institute. We thank Dr. Marion Wassef (École Normale Supérieure, Paris, France) and F. Qiu (University of Medicine and Dentistry of New Jersey, Piscataway, NJ) for giving us various (DNAs.

Correspondence should be addressed to Dr. Alain Chédotal, Institut National de la Santé et de la Recherche Médicale, Unité Mixte de Recherche en Santé 592, Institut de la Vision, F-75012 Paris, France. E-mail: alain.chedotal@inserm.fr.

K. T. Nguyen-Ba-Charvet's and C. Sotelo's present address: Institut National de la Santé et de la Recherche Médicale, Unité Mixte de Recherche en Santé 592, Institut de la Vision, F-75012 Paris, France.

DOI:10.1523/JNEUROSCI.0078-08.2008

Copyright (C) 2008 Society for Neuroscience $\quad 0270-6474 / 08 / 286285-10 \$ 15.00 / 0$ netrin-1/deleted in colorectal cancer (DCC) and slit/roundabout (Robo) (Dickson, 2002; Dickson and Gilestro, 2006).

In vertebrates, hindbrain precerebellar neurons represent an excellent system for studying the regulation of midline crossing. They all originate from the rhombic lip, a dorsal stripe of neuroepithelium lining the edges of the fourth ventricle (Wingate, 2001). Inferior olivary neurons (IO neurons) are the source of the climbing fibers and synapse on Purkinje cell dendrites in the cerebellar cortex. All other hindbrain precerebellar neurons, the lateral reticular nucleus (LRN), the external cuneatus nucleus (ECN), and the pontine neurons (nucleus reticularis tegmenti pontis and basal pontine gray) (PN), send mossy fibers on granule cell dendrites. During development, hindbrain precerebellar neurons migrate tangentially from the rhombic lip toward the floor plate (Bourrat and Sotelo, 1990a,b; Kawauchi et al., 2006). On reaching the floor plate, they exhibit distinct behaviors: IO neurons stop and only their axons cross, LRN and ECN neurons cross entirely, and most PN neurons stop but some also cross (Fig. 1). Netrin-1 was shown to attract all precerebellar neurons toward the midline (Bloch-Gallego et al., 1999; Yee et al., 1999; Causeret et al., 2002). We showed previously that the receptor Rig1/Robo3 (hereafter referred to as Robo3) has a major function in this process (Marillat et al., 2004) because precerebellar neurons are unable to cross the midline in $R o b o 3^{-1-}$ mutant embryos and send their axons into the ipsilateral cerebellum. Robo3 plays a similar role in spinal cord commissural axons (Sabatier et al., 2004). The current working model is that Robo3 somehow interferes with Slit-mediated repulsion, thereby allowing Robo1/ 


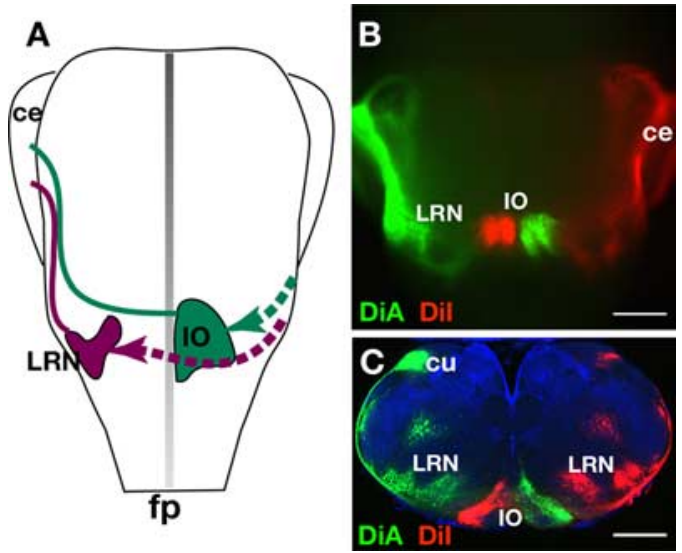

Figure 1. Organization of $\mathrm{IO}$ and LRN projections. $A$, Schematic representation of the migration pathways (dashed lines) and projections of I 0 neurons (green) and LRN neurons (purple) to the cerebellum (ce). These neurons come from the rhombic lip and migrate ventrally toward the floor plate (fp). The cell bodies of $I 0$ neurons stop at the ventral midline, whereas LRN neuron cross it. However, 10 axons also cross the midline. $\boldsymbol{B}, \boldsymbol{C}$, Whole mount ( $\boldsymbol{B}$ ) (ventral side) or coronal section $(\boldsymbol{C})$ of E18 mouse embryos injected with DiA [4-(4-dihexadecy-lamino)-styryl)- $N$ methylpyridinium iodide] in the right side of the cerebellum and Dil in the left side. 10 neurons are labeled on the controlateral side of the injection and LRN on the ipsilateral side. Scale bars: $B, 740 \mu \mathrm{m} ; C, 500 \mu \mathrm{m}$

2-expressing axons to reach and cross the midline (Long et al., 2004; Sabatier et al., 2004). To test this model, we analyzed here the development of posterior precerebellar neurons (IO, LRN, and ECN) in mice deficient for Slit1 and Slit2 or Robo receptors (Plump et al., 2002; Grieshammer et al., 2004; Long et al., 2004).

\section{Materials and Methods}

Mice. Swiss mice (Janvier) were used for expression studies. Slit-deficient mice and Robo-deficient mice (all in C57BL/6 inbred strain) were described previously and genotyped by PCR (Plump et al., 2002; Grieshammer et al., 2004; Long et al., 2004; Sabatier et al., 2004; Fouquet et al., 2007). The day of the vaginal plug was counted as embryonic day 0 (E0), and the day of the birth as postnatal day 0 (P0).

Immunocytochemistry. Until E16, embryos were fixed by immersion in $4 \%$ paraformaldehyde in $0.12 \mathrm{~m}$ phosphate buffer, $\mathrm{pH} 7.4$ (PFA). Older mice were perfused transcardially with $4 \%$ PFA, postfixed for $3 \mathrm{~h}$, and then cryoprotected in $10 \%$ sucrose. Cryostat sections were blocked in $0.2 \%$ gelatin in PBS containing $0.25 \%$ Triton X-100 (Sigma-Aldrich) and incubated overnight at room temperature with rabbit anti- $\beta$ galactosidase (MP Biomedicals), rabbit anti-green fluorescent protein (GFP) (Invitrogen), chicken anti-GFP (Abcam), rabbit anti-Brn3.2 (Millipore Bioscience Research Reagents), rabbit anti-Pax6 (Millipore Bioscience Research Reagents), mouse anti-Calbindin ${ }_{\mathrm{D} 28 \mathrm{~K}}$ (Swant), goat anti-ALCAM (activated leukocyte-cell adhesion molecule)/BEN (R\&D Systems), followed by species-specific secondary antibodies directly conjugated to fluorophores (Jackson ImmunoResearch or Invitrogen). Sections were examined under a fluorescent microscope (DMR6000; Leica) or a confocal microscope (SP5; Leica).

In situ hybridization. Antisense riboprobes were labeled with digoxigenin-11-D-UTP (Roche Diagnostics) as described previously (Marillat et al., 2002), by in vitro transcription of mouse or rat cDNAs encoding Slit1, Slit2, Slit3, Robo1, Robo2 (Brose et al., 1999), Robo3 (Marillat et al., 2004), Brn3.2, TAG-1, Pax6 (de Diego et al., 2002), Barhl1 (Li et al., 2004), and Er81 (Zhu and Guthrie, 2002). Tissue sections and whole-mount embryos were hybridized with digoxigenin-labeled riboprobes as described previously (Marillat et al., 2002, 2004).

1,1'-Dioctadecyl-3,3,3',3'-tetramethylindocarbocyanine injection. After intracardiac perfusion with $4 \%$ PFA, a small occipital craniotomy was performed to expose the cerebellum of E18-P0 mice. A small crystal of lipophilic tracer 1,1'-dioctadecyl-3,3,3',3'-tetramethylindocarbocyanine (DiI) (Invitrogen) attached to the tip of a broken glass pipette was applied on one side of the cerebellum. After $2-3$ weeks at $37^{\circ} \mathrm{C}$ in the dark, the brain was dissected out and photographed. Some injected brains were also embedded in $2 \%$ agarose, and cut in $200-\mu \mathrm{m}$-thick sections with a vibratome (Leica). The sections were then counterstained in a solution of $10 \mu \mathrm{g} / \mathrm{ml}$ Hoechst 33258 (Sigma-Aldrich) in PBS for $30 \mathrm{~min}$, rinsed three times in PBS, and stored in $4 \%$ PFA.

Statistical analysis. To quantify the number of IO neurons entering the midline, three series of 20 - $\mu \mathrm{m}$-thick sections were obtained from E13 embryos hindbrain. One series was double labeled with Brn3.2 and Pax6, counterstained with Hoechst, and used for counting.

On each section of the series containing olivary neurons, the number of Brn3.2-positive/Pax6-negative neurons in a 45 - $\mu \mathrm{m}$-wide region centered on the midline was counted using MetaMorph software (Molecular Devices). Series from three to five animals of each genotype were counted. For statistical analysis, we used a paired $t$ test (GraphPad Software) to compare two conditions (wild type/other genotype). Compiled data are expressed as mean \pm SEM.

\section{Results}

\section{Expression of Robo receptors in migrating precerebellar neurons}

Using in situ hybridization, we first showed that IO neurons and LRN neurons expressed mRNA encoding Robo1, Robo2, and Robo3 at E12-E13, during their migration from the rhombic lip (Fig. 2A-C) (Marillat et al., 2002, 2004). As previously described, Robo3 was rapidly downregulated after their leading processes crossed the floor plate (Fig. 2C) (data not shown) (Marillat et al., 2004). In Robol-deficient mice, a cassette encoding $\beta$-galactosidase was inserted in the Robol locus (Long et al., 2004). Similarly, $\beta$-galactosidase-fused with tau was inserted into the Robo2 locus (Grieshammer et al., 2004). In E13-E15 Robo $1^{-1-}$ mice, $\beta$-galactosidase immunoreactivity (restricted to intracytoplasmic vesicles) was detected in migrating IO neurons that expressed the transcription factor Brn3.2 (Fig. 2D) (McEvilly et al., 1996), and in LRN neurons, which were not labeled by Brn3.2 antibodies (data not shown). Likewise, these neurons also expressed $\beta$-galactosidase in E13-E15 Robo2 ${ }^{-1-}$ mice, but in this case, the whole cell was labeled (Fig. $2 \mathrm{~F}-H$ ). We could also confirm that mRNAs encoding the three mouse slit genes (Slit1-Slit3) were expressed by floor plate cells in the hindbrain during precerebellar neuron migration (supplemental Fig. 1, available at www.jneurosci.org as supplemental material).

\section{Abnormal migration of IO neurons across the floor plate in Robo mutants}

We showed previously that all precerebellar neurons and axons are unable to cross the ventral midline in mice deficient for Robo3 (Marillat et al., 2004) (see below). By analogy with the current model of Robo3 function in spinal cord commissural axons (Sabatier et al., 2004), we proposed that this phenotype was attributable to a premature activation of Robo1 and Robo2 receptors by floor plate-derived slits. The validation of this hypothesis first requires showing that Robo1 and Robo2 mediate a repulsive Slit signal for precerebellar neurons. To test this model, we analyzed the phenotype of Robo1, Robo2, Slit1, and Slit2 single and double knock-out mice. As shown previously, our Robol allele is likely to be a severe hypomorph rather than a complete null (Long et al., 2004; Lopez-Bendito et al., 2007). IO neurons were visualized using Brn3.2 immunostaining (Fig. $3 A-K$ ). LRN neurons were identified using Pax6 immunostaining and Hoechst staining (Fig. $3 B, C$ ) (Engelkamp et al., 1999). These two types of neurons are known to migrate tangentially close to the pial surface of the caudal hindbrain following two parallel and independent routes, just under the pial surface for LRN neurons, and deeper in the 


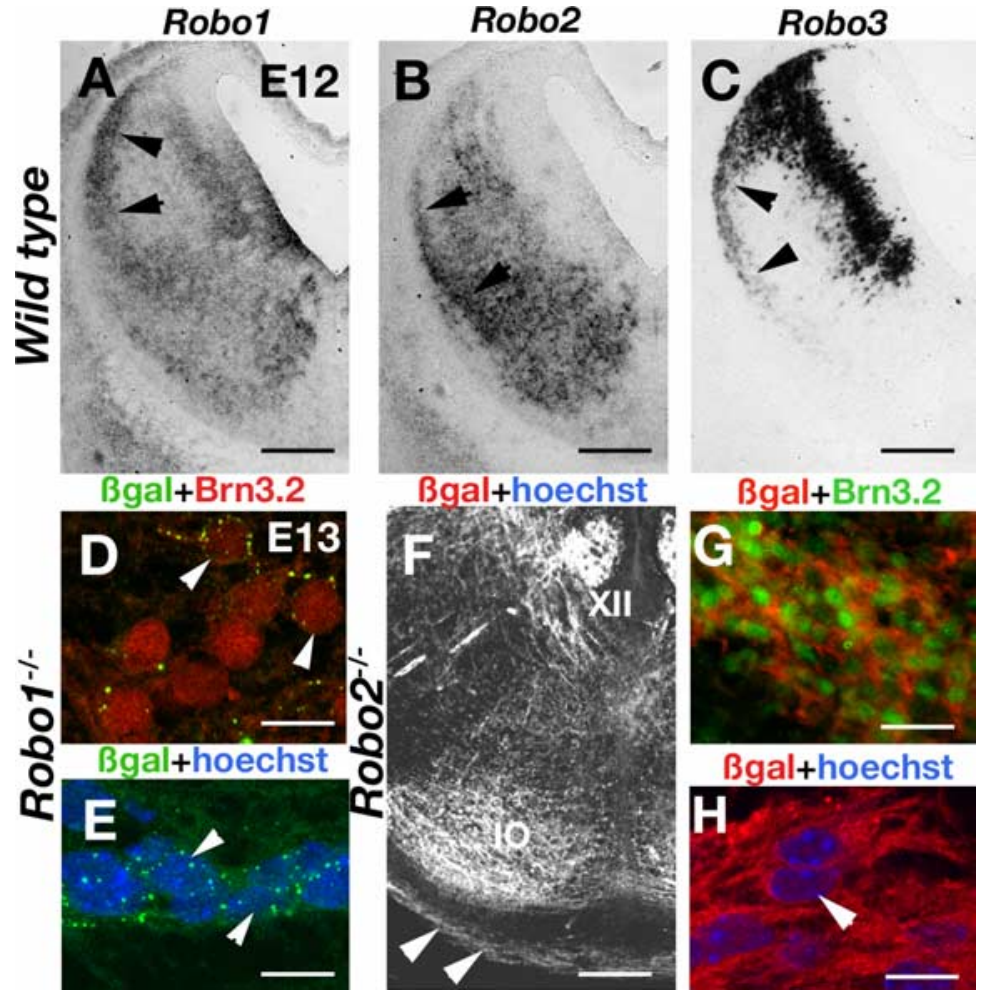

Figure 2. Expression of Robo receptors in migrating 10 and LRN neurons. $\boldsymbol{A}-\boldsymbol{C}$, Consecutive coronal sections of E12 mouse embryos hybridized with digoxygenin-labeled riboprobes for $\operatorname{Rob01}(\boldsymbol{A}), \operatorname{Rob02}(\boldsymbol{B})$, and $\operatorname{Rob03}(\boldsymbol{C})$. Precerebellar neurons leaving the rhombic lip express the three mRNAs (arrowheads). $\boldsymbol{D}, \boldsymbol{E}$, Three micrometer coronal sections of E13 Robo $1^{-/-}$embryos. Punctate $\beta$-galactosidase immunoreactivity is detected both in Brn3.2-immunopositive 10 neurons ( $\boldsymbol{D}$, arrowheads) $(3 \mu \mathrm{m}$ confocal image) and in LRN neurons (visualized by Hoechst staining) ( $\boldsymbol{E}$, arrowheads) (3 $\mu \mathrm{m}$ confocal image). $\boldsymbol{F}$ - $\boldsymbol{H}$, Coronal sections of E13 Rob02 $2^{-1-}$ embryos. Diffuse $\beta$-galactosidase immunoreactivity is detected in 10 neurons (IO in $\boldsymbol{F}$ ) and the marginal stream of migrating LRN neurons ( $\boldsymbol{F}$, arrowheads). $\beta$-Galactosidase is coexpressed with Brn3.2 in 10 neurons $(\boldsymbol{G})$ and in the stream of LRN neurons visualized with Hoechst ( $\boldsymbol{H}$, arrowhead) (3 $\mu \mathrm{m}$ confocal image). Scale bars: $\boldsymbol{A}-\boldsymbol{C}, 170 \mu \mathrm{m} ; \boldsymbol{D}, 8 \mu \mathrm{m} ; \boldsymbol{E}$, $20 \mu \mathrm{m} ; \boldsymbol{F}, 100 \mu \mathrm{m} ; \boldsymbol{G}, 26 \mu \mathrm{m} ; \boldsymbol{H}, 10 \mu \mathrm{m}$. XII, Hypoglossal motor nucleus.

brain parenchyma for IO neurons (Fig. $3 A, B$ ). Whereas LRN neurons cross the floor plate to settle on the opposite side of the brain, IO neurons are unable to cross it (Fig. 1C). This distinct behavior could be seen using Brn3.2 and Hoechst double staining. In our working model, Robol and Robo2 cooperate to prevent the cell bodies of IO neurons from crossing the midline. We first quantified the number of Brn3.2 IO neurons encountered in the floor plate of wild type, Slit1/2 and Robo1/2 knock-outs, at E13, when the bulk of IO neurons reaches the midline (Fig. 3A$K)$. The number of IO neurons found in the floor plate was extremely low in wild type $(n=5)$ as well as in Robo1 ${ }^{+/-}(n=3)$, Robo1 $^{-/-}(n=4)$, Robo $^{+/-}(n=5)$, and Slit1 ${ }^{-/-}(n=3)$ embryos (Fig. $3 D-F, H, L$ ) (data not shown). In contrast, there was a significant increase of the number of IO neurons in the midline of Robo2 ${ }^{-/-}(n=4$ of 4$)$, Slit $^{-1-}(n=5$ of 5$)$, and Slit $1^{-1-} ;$ Slit $2^{-1-}(n=3$ of 3$)$ mutant embryos, which reached a maximum in Robo1 ${ }^{-1-} ;$ Robo2 $^{-1-}(n=4$ of 4$)$ double mutants (Fig. 3I-L). In all these knock-outs, midline crossing by LRN neurons was always observed as in wild-type embryos (Fig. 3). This result suggested that, in these mutants, IO neurons were less sensitive to midline-derived Slits and may cross the ventral midline. To further confirm that some IO neurons may cross the midline in Slit and Robo knock-outs, DiI was injected unilaterally in the cerebellum of E18-P0 animals (Marillat et al., 2004). In all wild type $(n=5), \operatorname{Robo1}^{-/-}(n=5), \operatorname{Slit1}^{-/-}(n=2)$, and Slit $^{+/-}(n=2)$ mice, a retrograde tracing of the inferior olivary nucleus located on the contralateral side was observed (Fig. 4A,D) (data not shown). IO axons could be visualized as they crossed the floor plate in the interolivary commissure. In contrast, an increasing number of DiIlabeled IO neurons was observed on the injection side in all Robo2 $2^{-/-}(n=3)$, Slit $2^{-/-}(n=3)$, Slit1 ${ }^{-/-} ;$Slit2 ${ }^{-/-}(n=5)$, and Robo1 ${ }^{-1-} ;$ Robo2 $^{-1-}(n=8)$ mutant mice (Fig. $4 B, C, E, F$ ). The ipsilateral DiIlabeled IO neurons were found in all IO subdivisions, with some variability between cases. Their proportion (determined by counting, on $5 \mu \mathrm{m}$ confocal images, the number of DiI-labeled cells on each side) varied between 10 and $\sim 40 \%$ of the labeled neurons in Robo1 $^{-1-} ; R_{0 b o} 2^{-1-}$ embryos (Fig. $4 F$ ). This difference between animals may be attributed to the variability of the DiI tracing efficiency and injection sites between animals, and does not provide an exact value of the total number of ipsilateral/contralateral IO neurons. This could suggest that, in these mutants, some IO axons failed to cross the midline and projected ipsilaterally (a hypothesis that would argue against a repulsive activity of Slit/Robo). However, we rather propose that the ipsilateral IO projection is attributable to aberrant midline crossing by some IO neurons, an interpretation supported by the significant increase of Brn3.2positive IO neurons in the mutants floor plate.

\section{Abnormal morphology and lamellation of the inferior olive in Slit and Robo knock-outs}

In wild-type mice, the transcription factors Brn3.2 and Er81 (Zhu and Guthrie, 2002; Marillat et al., 2004) are strongly expressed in specific subdivisions of the inferior olivary complex, symmetrically organized on both sides of the floor plate. Three main IO subdivisions can be recognized in all vertebrate species (Azizi and Woodward, 1987), the medial accessory olive (MAO), the dorsal accessory olive (DAO), and the principal olive (PO) (Fig. 5). These main subdivisions can be further divided into smaller entities (subnuclei). For instance, the MAO comprises a horizontal, a vertical, and a rostral lamella; the DAO is subdivided into dorsal and ventral folds; and the PO into ventral and dorsal lamellas. IO neurons in each of these subnuclei project to specific sagittal stripes within the vermal and hemispheric cortices (Azizi and Woodward, 1987). Brn3.2 is expressed by all IO neurons but at different intensities depending on their subnuclear location: the lateral part of the ventral fold of the DAO, the medial one-half of the dorsal lamella of the PO, and the ventral part of the central MAO are those with the highest expression. In contrast, Er81 is highly, also unevenly expressed in the DAO and PO but is excluded from the MAO (Fig. 5G). The use of these transcription factors, combined with other IO neuron markers [calciumbinding protein calbindin D28K (CaBP), BEN] allows an almost perfect identification of most of the olivary subnuclei in wildtype and knock-out mice. In Slit1/Slit2 and Robo1/Robo2 single and double mutants, all IO neurons were still able to migrate ventrally on both sides of the floor plate (no ectopic neurons were 
found along the migration route), but the overall morphology of the nuclear complex was modified. In situ hybridization with Er81 riboprobes showed that, in E18 embryos, the IO was more compact and closer to the midline in Slit $2^{-/-}(n=2$ of 2 ) and Robo2 ${ }^{-1-}(n=5$ of 5$)$ single mutants or in Slit $1^{-/-} ;$Slit2 $2^{-1-}(n=4$ of 4$)$ and Robor $^{-1-} ;$ Robo $^{-1-}(n=6$ of 6$)$ double mutants than in wild type, Slit1 ( $n=3$ of 3 ), and Robol ( $n=6$ of 6 ) single mutants or heterozygous mice (Fig. $5 A-$ $D, G-I)$ (data not shown). The increased mediolateral compaction of the IO nucleus was also observed in embryos labeled with Brn3.2 probes or antibodies (Fig. $5 E, F, J-L)$. This abnormal structure of the IO complex was even more striking on coronal sections from Slit1 ${ }^{-/-}$;Slit2 ${ }^{-/-}$ and Robo1 ${ }^{-/-}$;Robo2 $2^{-/-}$mutants labeled with Brn3.2 or Er81. First, the dorsal lamella of the DAO was more compact, more dorsal, and abnormally curved toward the floor plate, and second, the MAO was interwoven with the PO (Fig. 5G-M). Despite these important cytoarchitectonic changes, neurons from the different IO subdivisions were apparently not mixed, as shown using immunostaining for Brn3.2 and CaBP (Marillat et al., 2004) (supplemental Fig. 2, available at www.jneurosci.org as supplemental material). Likewise BEN-positive IO neurons were still clustered (data not shown). At E18, Robo2 mRNA was more highly expressed in the DAO and dorsal MAO (supplemental Fig. 2, available at www.jneurosci.org as supplemental material). Robo2 in situ hybridization on sections from Slit1/2 double mutants confirmed the abnormal compaction of the DAO (supplemental Fig. 2, available at www.jneurosci.org as supplemental material). These results show that the final positioning of IO neurons and particularly the lamellation of the inferior olive are at least partially controlled by Slit/Robo signaling.

\section{Phenotypic changes in brainstem mossy fiber neurons (LRN/ ECN) in Slit and Robo knock-outs}

We next examined whether LRN/ECN neurons were also affected by the absence of Slit1/2 or Robo1/2 using in situ hybridization for Barhl1, a transcription factor strongly expressed by these neurons (Li et al., 2004). The position of the LRN was still comparable with wild type in the double Slit $1^{-/-} ;$Slit $2^{-/-}(n=10$ of 10$)$ and Robo1 ${ }^{-1-}$;Robo2 ${ }^{-1-}$ mutants ( $n=9$ of 9 ), although the nucleus was thinner and its size apparently reduced (Fig. 6). Likewise, ECN neurons were found at their normal dorsal location, caudal to the cerebellum (Fig. 7) but in their case, the organization/morphology of their nuclei was very perturbed, especially in double mutants. Whereas the ECN morphology did not seem affected in Slit1 ${ }^{-/-}(n=4)$ and Robo1 ${ }^{-1-}(n=4)$ single mutants (Fig. 7), the ECN was less compact and more elongated caudally in Slit2 ${ }^{-1-}(n=3$ of 3$)$ and $\operatorname{Robo2}^{-1-}(n=6$ of 6$)$ mutants. This caudal extension of the ECN associated with a fragmentation of the nucleus was much more pronounced in Slit ${ }^{-1-} ;$ Slit $2^{-1-}(n=10$ of 10$)$ and Robo1 ${ }^{-1-} ;$ Robo $^{-1-}(n=$ 9 of 9 ) double mutants (Fig. 7). These results confirm that the migration of hindbrain precerebellar neurons is controlled by Slit/Robo signaling.

\section{Migration of LRN and IO neurons in Robo1/2/3 triple mutants}

The current working hypothesis of Robo3 function in hindbrain and spinal cord commissural projections is a negative regulation of Robo1/2-mediated Slit repulsion before midline crossing. In this model, Robo3 somehow prevents Slits from activating Robo $1 / 2$ receptors during axonal and neuronal migration toward the floor plate. Accordingly, we showed that Robo3-expressing precerebellar axons or neurons are unable to cross the ventral 
Slit1+1/;Slit2+1-
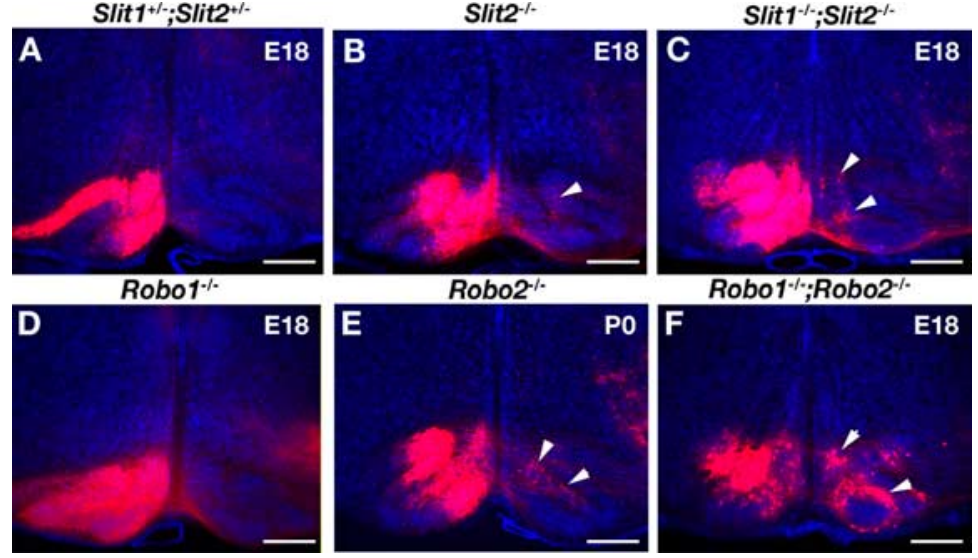

Figure 4. Ipsilateral I0 projection in Slit and Robo mutants. Coronal vibratome sections of E18 embryos injected unilaterally into the cerebellum with Dil and counterstained with Hoechst. $\boldsymbol{A}-\boldsymbol{F}, \mathbf{I 0}$ neurons are retrogradely labeled on the controlateral side in Slit1 ${ }^{+/-} ;$Slit2 $2^{+/-}(\boldsymbol{A})$ and Robo1 $1^{-/-}(\boldsymbol{D})$ embryos but an increasing number of Dil-labeled 10 neurons are found on the

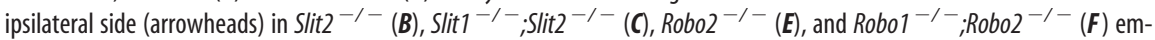
bryos. Scale bars: $\boldsymbol{A}-\boldsymbol{F}, 120 \mu \mathrm{m}$.

midline in Robo3 $^{-1-}$ mutants (Marillat et al., 2004) (see also below) and that many IO neurons abnormally cross it in Robo1 ${ }^{-/-} ;$Robo2 $2^{-1-}$ double mutants. To try to further confirm this model, we generated Robo1 ${ }^{-/-} ; R_{0 b o 2^{-/-}} ;$Robo3 $^{-/-}$triple mutants (hereafter referred to as Robo1/2/3 triple mutants) and analyzed precerebellar neuron migration and axonal projections. As described previously (Marillat et al., 2004; Sabatier et al., 2004), GFP was inserted in the robo3 locus and was used to identify Robo3-expressing precerebellar neurons at E13 in Robo3 knock-outs, as they reach the floor plate. Migrating IO neurons and LRN neurons can be clearly distinguished in $\mathrm{Robo3}^{+/-}$mice by double immunostaining for GFP and Brn3.2 (Fig. 8 A, B). Only Brn3.2-negative/GFP-positive LRN neurons cross the floor plate (Fig. $8 A, B$ ). The stream of LRN neurons crossing the floor plate could also be visualized with in situ hybridization for Barhl1 (Fig. $8 C, D)$. As described above for Robo1 ${ }^{-/-} ;$Robo $^{-/-}$double mutants, many IO neurons (Brn3.2 positive/GFP positive) crossed the floor plate in $\mathrm{Robo1}^{-/-} ; \mathrm{Robo2}^{-/-} ; \mathrm{Robo3}^{+/-}$mice $(n=2$ of 2) (Fig. $8 E, F$ ), in addition to LRN neurons (Fig. $8 E-H$ ). In contrast, no GFP processes or neurons were observed in the midline of E13 Robo3 ${ }^{-1-}$ mutants ( $n=3$ of 3 ), and both LRN and IO neurons were found at an abnormal distance from the floor plate (Fig. $8 I-L$ ). In Robo1/2/3 triple mutants ( $n=2$ of 2 ), IO neurons were still able to form a nucleus near the ventral midline but no Brn3.2-positive neurons were found inside the floor plate (Fig. $8 M, N$ ) similarly to wild type and Robo3 single mutants but in contrast to Robo1/2 double mutants. Although the number of cell bodies of LRN neurons at the midline was reduced in E13 Robol/ 2/3 triple mutants (Fig. 8M-O), many GFP- or Barhl1-positive cells were found in the midline. By E15, the stream of crossing LRN neurons was clearly visible using Barhl1 in situ hybridization (Fig. $8 P)(n=1)$. This suggests that, in contrast to IO neurons, the Robo3 LRN migration defect is partially rescued in Robo1/2/3 triples.

The similarity between the IO axon midline crossing defects in Robo1/2/3 triple mutants and Robo3 single mutants was confirmed by performing unilateral DiI injection into the cerebellum. In both Robo3 single mutants ( $n=8$ of 18) and Robol/2/3 triple mutants ( $n=2$ of 2 ), DiI-labeled IO axons did not cross the floor plate and projected ipsilaterally (Fig. 9A-D), instead of the opposite side as in wild-type animals (compare with Fig. 4A).
Therefore, these observations suggest that Robo3 function in the IO nucleus is not merely to regulate Slit/Robo1/Robo2 inhibition and may even be dominant over Robo1/Robo2 signaling. Coronal sections of the inferior olive of E18 Robo1/2/3 triple mutants labeled with $E r 81$ probe $(n=2$ of 2), or Brn3.2 and CaBP antibodies (supplemental Fig. 2, available at www.jneurosci.org as supplemental material), showed that IO lamellation was more severely perturbed than in Robo3 single mutants (Fig. $7 E, F)$, thereby suggesting that the three receptors cooperate to control IO nucleogenesis.

\section{Discussion}

\section{Slit/Robo interactions prevent IO} neurons from crossing the midline

His (1890) first proposed that, in human embryos, inferior olivary neurons are generated dorsally in the rhombic lip and migrate ventrally (Altman and Bayer, 1978). This observation was confirmed experimentally in chick embryos (Harkmark, 1954) and by birthdating studies in rodents (Ellenberger et al., 1969; Altman and Bayer, 1978, 1985). Slice culture experiments showed that tangentially migrating inferior olivary neurons send a long leading process across the midline but that their cell body always stays ipsilateral (Bourrat and Sotelo, 1988) (Fig. 10). The leading process of IO neurons transforms into an axon that approaches the cerebellum as the cell body reaches to the floor plate (Wassef et al., 1992). The floor plate was then proposed to attract ventrally migrating precerebellar neurons (Bourrat and Sotelo, 1990b), a process later shown to involve DCC/netrin-1 (Bloch-Gallego et al., 1999; Yee et al., 1999; Causeret et al., 2002). However, the molecular mechanism that stops IO cell bodies at the floor plate is unknown.

Slit/Robo signaling controls midline crossing by a variety of axons (Dickson, 2002; Fouquet et al., 2007). In Drosophila, Slit/ Robo signaling also prevents tracheal, sensory precursor cells, and cardiac cells from approaching the midline (Lundstrom et al., 2004; Orgogozo et al., 2004; Santiago-Martinez et al., 2006). In vertebrates, a similar function for Slit/Robo in the control of neuronal migration at the midline has not been demonstrated. We show here that IO neurons can cross the floor plate in Slit and Robo mutants (Fig. 10). These results support a simple model according to which the binding of midline-derived Slits to IO neurons forces their cell bodies to stay on the ipsilateral side and pushes their axons away from the floor plate after crossing. Although this phenotype is stronger in Robo1/2 double mutants, some IO neurons also cross in Robo2 single mutants. Therefore, Robo2 appears to have the strongest repulsive activity in this system, but Robo1 cooperates with Robo 2 to prevent crossing. However, the Robol knock-out line used here was obtained by gene trap method and may be only be a hypomorphic allele (Long et al., 2004; Fouquet et al., 2007). Accordingly, their telencephalon has a milder phenotype than another line obtained by classic gene targeting method (Andrews et al., 2006; Lopez-Bendito et al., 2007). It would be important to compare the IO development of the two Robol lines. Although, in many types of commissural axons, Robo1 and Robo2 were also shown to act redundantly (Fouquet et al., 2007; Lopez-Bendito et al., 2007), Robol is the major repulsive receptor in spinal cord commissural axons (Long 
et al., 2004). Our data suggest that Robo2mediated repulsion prevails in IO neurons over Robol. The IO neuron crossing defects are also stronger in Slit1/2 double mutants than in the Slit1 and Slit2 single mutants but more moderate than in Robol/2 double mutants. This suggests that some floor plate repellent, most likely Slit3, is still detected by IO neurons, as previously observed in the spinal cord (Long et al., 2004).

One important remaining issue is to understand the cellular and molecular mechanisms downstream of Robo receptors that can selectively block the translocation of IO nucleus before the floor plate. Using the available anti-Robol and -Robo 2 antibodies, we were not able to determine whether their cell membrane distribution is compartmentalized along IO axons, as described for Drosophila commissural axons (Kidd et al., 1998; Keleman et al., 2005) (T. Di Meglio and A. Chédotal, unpublished data).

\section{Slit/Robo signaling and IO lamellation}

In Slit and Robo mutants, the IO complex is disorganized: although neurons are able to reach the ventral midline, the morphology of the three main IO subdivisions is abnormal. In the Robo1/2 double mutants, a fraction of olivary neurons (that do not appear to belong to specific of IO subnuclei) migrate across the midline, and as a consequence, the two inferior olives are composed of a mixed population of neurons originating from both sides. Although the cytoarchitecture of these nuclei is somewhat disrupted, the typical clustering of inferior olivary subnuclei appears to be preserved as shown using markers of IO subsets, suggesting that neurons from the two different sides may still preferentially cluster. However, the different IO subdivisions are almost impossible to delimit in Robo1/2/3 triple mutants. The aggregation of IO neurons and the formation of the distinct lamella is a late process and the position of IO neurons depends on their birthdate (Bourrat and Sotelo, 1991). DAO neurons are born first and are also the ones that are the most affected in Slit/Robo mutants. Before IO subdivisions adopt their final morphology, they already express different combination of proteins (Wassef et al., 1992), including axon guidance molecules and receptors (Chedotal et al., 1996; Nishida et al., 2002; Backer et al., 2007). Interestingly, mice lacking the RhoGEF (guanine exchange factor) Trio exhibit IO lamellation defects resembling those observed in Robo1/Robo2 and Slit1/Slit2 mutants (Backer et al., 2007). Neurons of the DAO that are the most severely affected were shown to strongly express Cadherin-11 (Backer et al., 2007), but also Robo2 (this study). Robo was previously found to inhibit N-cadherin-mediated adhesion (Rhee et al., 2002), and in Caenorhabditis elegans, UNC73, the TRIO homolog, binds and modulates the repulsive activity of SAX-3 (Watari-Goshima et al., 2007). This suggests that, in addition to controlling midline crossing, Robo receptors influ- ence IO nucleogenesis via homophilic and heterophilic interactions (Hivert et al., 2002; Camurri et al., 2005) or by modulating cadherin-mediated cell adhesion. This could also be the case for LRN and ECN neurons whose migration is also controlled by cadherins (Taniguchi et al., 2006) and are less compact in absence of Slit/Robo signaling.

\section{A distinct function for Robo3 in LRN/ECN neurons and IO neurons}

We previously proposed that LRN and ECN neurons are repelled by Slits after crossing the midline (Marillat et al., 2004). We bring here direct in vivo evidence suggesting that Slit/Robo signaling controls the final positioning of LRN and ECN neurons (Fig. 10). Moreover, midline crossing by LRN neurons appears at least partially rescued in the absence of Robol and Robo2, which is consistent with our initial model of action of Robo3 (Marillat et al., 2004), and its proposed function in spinal cord commmissural axons (Sabatier et al., 2004) (i.e., to suppress repulsion mediated by Robo1 and Robo2).

In the case of IO axons, however, the role of Robo3 appears to be different. As discussed above, our data support a role for Robo1 and Robo 2 in controlling midline crossing of both cell 

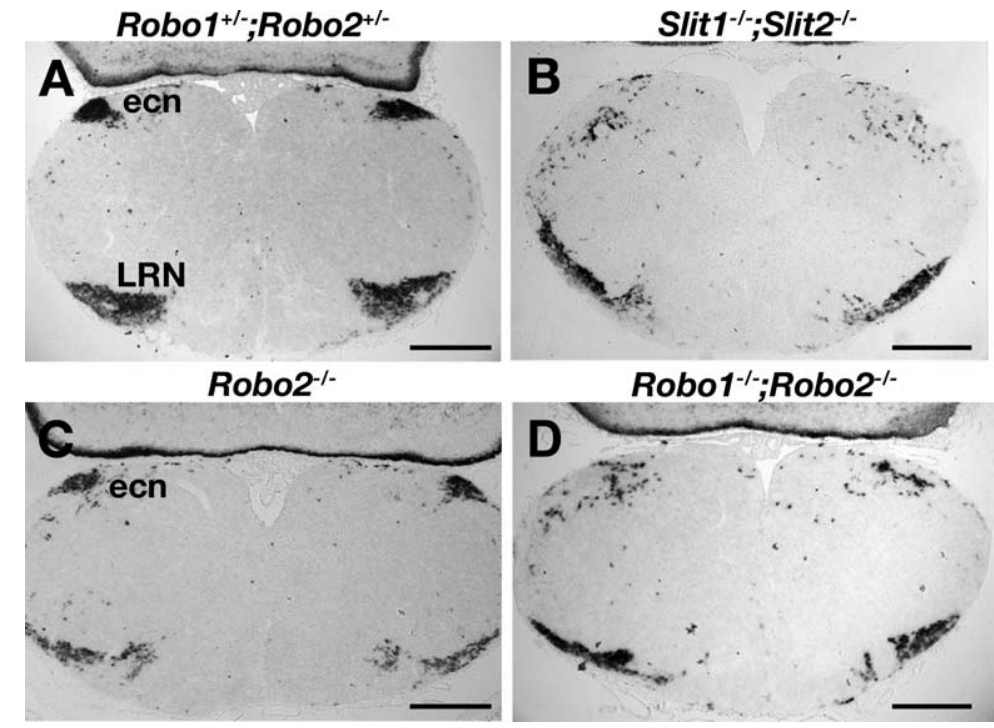

Robo1 $^{-/-; R o b o 2^{-/-}}$

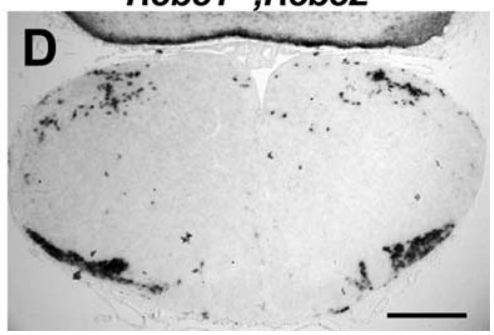

Figure 6. LRN defects in Slit and Robo mutants. $\boldsymbol{A}-\boldsymbol{D}$, Coronal sections at the level of the LRN of $\operatorname{Robo1}^{+/-} ; \operatorname{Robo2}^{+/-}(\boldsymbol{A})$, Slit1 ${ }^{-1-} ;$ Slit2 ${ }^{-1-}(\boldsymbol{B})$, Robo2 $^{-1-}(\boldsymbol{C})$, and Robo1 ${ }^{-1-} ;$ Robo2 $^{-1-}$ (D) E18 embryos hybridized with Barhl1 riboprobe. In all three homozygous mutants, the LRN is not as compact and ventral as in $R o b 01^{+\prime-} ;$ Robo2 $2^{+/-}$embryos. Note also the absence

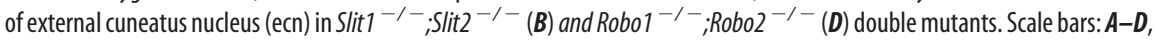
$520 \mu \mathrm{m}$.
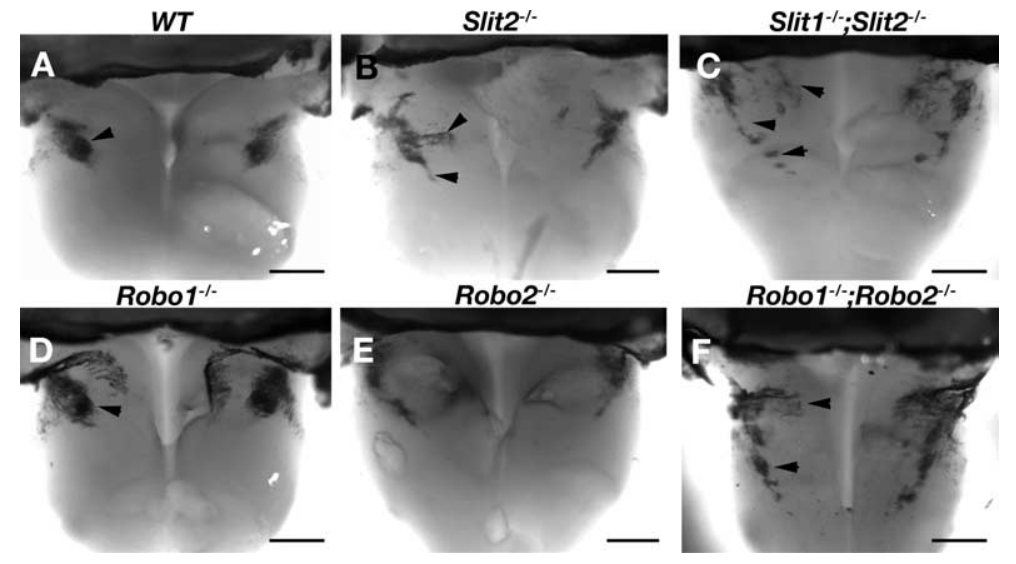

Figure 7. ECN defects in Slit and Robo mutants. $\boldsymbol{A}-\boldsymbol{F}$, Dorsal view of whole-mount hindbrains of E18 embryos hybridized with Barhl1 riboprobes. In wild type $(\boldsymbol{A})$ and $R o b 01^{-/-}$single mutant $(\boldsymbol{D})$, the external cuneatus nucleus (arrowhead) is a dorsal and ovoid nucleus located at a distance from the fourth ventricle just caudal to the rhombic lip. In Slit2 ${ }^{-1-}(\boldsymbol{B})$, Slit $^{-1-} ;$ Slit2 $^{-1-}$ $(\boldsymbol{C})$, Robo2 $^{-1-}(\boldsymbol{E})$, and Robo1 ${ }^{-1-} ;$ Robo2 $2^{-1-}(\boldsymbol{F})$, the ECN (arrowheads) is disorganized and does not form a well defined nucleus. It is also closer to the fourth ventricle and extends more caudally in the hindbrain. Scale bars: $A-F, 480 \mu \mathrm{m}$.

bodies and axons (Fig. 10). In wild type or Robo1/2 double mutants, IO axons cross the midline, but this crossing is blocked in Robo3 single mutants. Based on the standard model, we would expect this result to show that Robo3 normally suppresses repulsion mediated by Slit1/2. If that were the case, however, one might have expected removal of Robo1/2 in the Robo3 mutants to rescue midline crossing to some degree (a genetic suppression observed for commissural axons in the spinal cord) (Sabatier et al., 2004). Unexpectedly, in the Robo1/2/3 triple mutant, all IO axons fail to cross, as they do in the Robo 3 single mutant (Fig. 10). This provides genetic evidence that Robo3 may function independently of Robo1/Robo2 to guide IO axons at the midline and be absolutely required for crossing. Robo3 may silence some other inhibitory midline signaling system. Alternatively, Robo3 may function as an attractive receptor that enables midline crossing (a possibility that has not been excluded in the spinal cord) (Sabatier et al., 2004).
The effect of Robo3 on cell bodies of IO neurons is more difficult to establish conclusively. As discussed above, whereas the cell body of IO neurons cannot cross the floor plate in wild type, many cross it in Robo1/2 or Slit1/2 double mutants, consistent with the idea that Slit $1 / 2$ signaling via Robo1/2 prevents cell bodies from crossing the midline. In the Robol/2/3 triple mutants, the cell body of ION neurons did not cross (unlike what is seen in Robo1/2 double mutants), but this could simply reflect an indirect effect, because cell bodies would not be expected cross if the leading process they are moving in stay ipsilateral. For this reason, we favor a model in which Robo3 does not play any role in regulating the cell body migration of IO neurons, although the data do not exclude the possibility that Robo3 may play a positive role in enabling crossing, as we have postulated for the axons.

The distinct behavior of IO and LRN neurons at the midline may rely on a differential expression of axon guidance receptors. Accordingly, LRN neurons do not seem to express UNC5B receptors, contrary to IO neurons (Bloch-Gallego et al., 1999).

Likewise, the different types of precerebellar neurons may express distinct Robo3 isoforms. Several Robo3 splice variants have been described previously in vertebrates (Yuan et al., 1999; Jen et al., 2004; Camurri et al., 2005; Challa et al., 2005). Robo3 isoforms A and B differ in their N-terminal portion, which contains 26 additional amino acids in Robo3A (Camurri et al., 2005). These two isoforms seem to have distinct functions in zebrafish (Challa et al., 2005). More recently, it was shown (Chen et al., 2008) that there exists a Robo3.2 isoform differing from a Robo3.1 isoform in its extreme $\mathrm{C}$ terminus cytoplasmic domain. Interestingly, in spinal cord commissural axons, Robo3.1 is expressed before crossing and appears to block Robo1/2 repulsion, whereas Robo3.2 is expressed after crossing and cooperates with Robol and Robo2 to repel commissural axons from the floor plate.

Our results suggest that different precerebellar neurons use different molecular strategies to cross the midline.

\section{References}

Altman J, Bayer SA (1978) Prenatal development of the cerebellar system in the rat. II. Cytogenesis and histogenesis of the inferior olive, pontine gray, and the precerebellar reticular nuclei. J Comp Neurol 179:49-75.

Altman J, Bayer SA (1985) Embryonic development of the rat cerebellum. III. Regional differences in the time of origin, migration, and settling of Purkinje cells. J Comp Neurol 231:42-65.

Andrews W, Liapi A, Plachez C, Camurri L, Zhang J, Mori S, Murakami F, Parnavelas JG, Sundaresan V, Richards LJ (2006) Robol regulates the development of major axon tracts and interneuron migration in the forebrain. Development 133:2243-2252. 


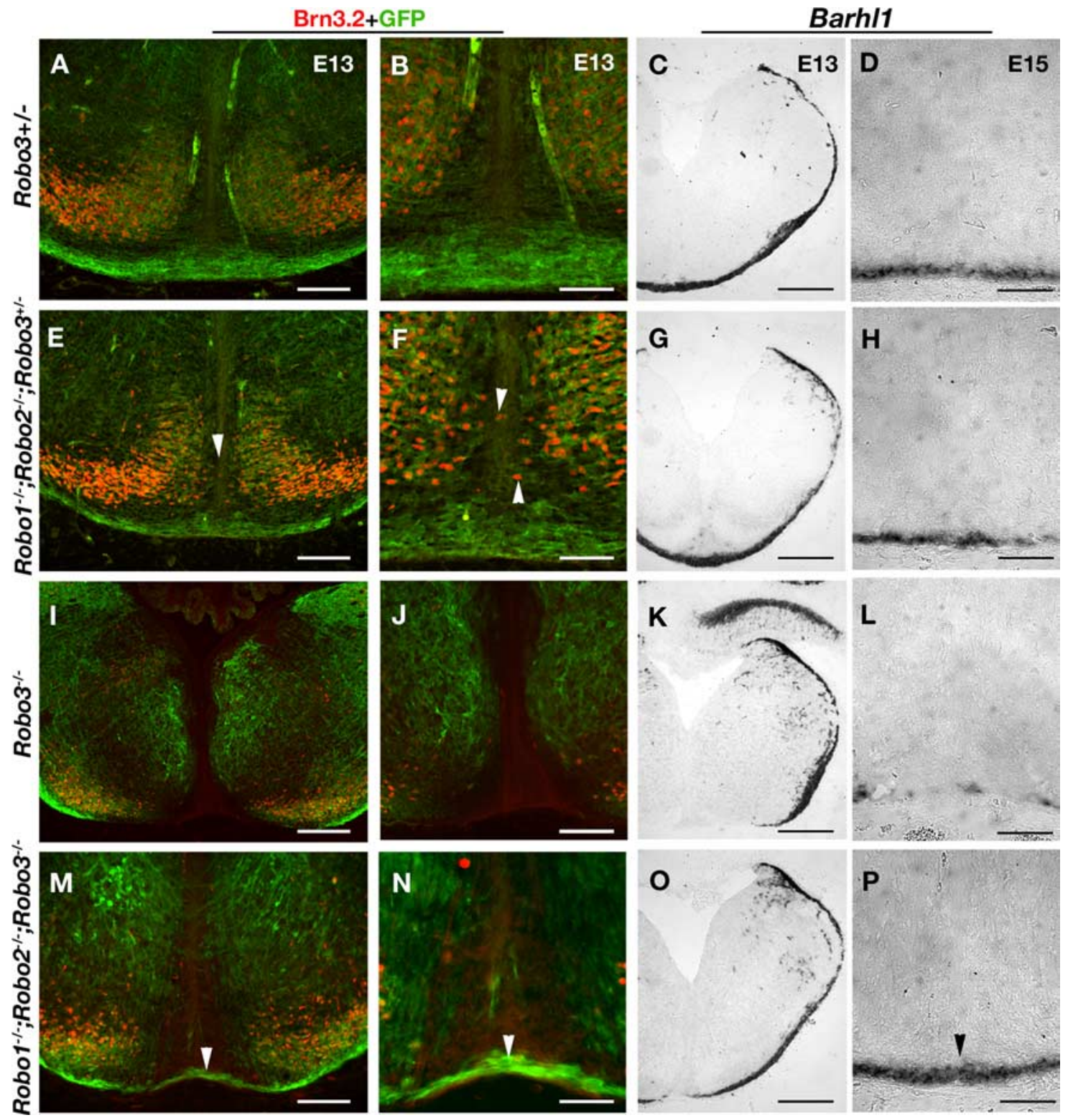

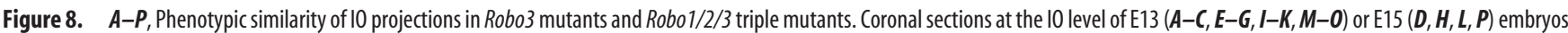

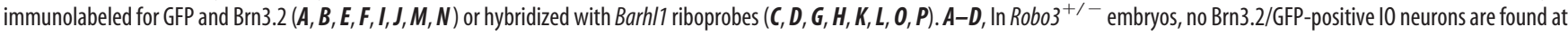
the midline, whereas Brn3.2-negative/GFP-positive and Barhl1-positive LRN neurons cross it. $\boldsymbol{E}-\boldsymbol{H}$, In contrast, many Brn3.2/GFP-positive I0 neurons (E, $\boldsymbol{F}$, arrowheads) are found in the floor plate

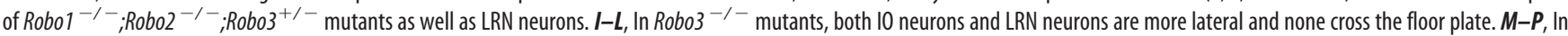
Robo $1 / 2 / 3$ triple mutants, 10 neurons do not enter the midline, but LRN neurons are closer from it than in Robo ${ }^{-1-}$ single mutants and many cross the midline $(M, N, P$, arrowheads). Scale bars: $A, E, M, 110 \mu \mathrm{m} ; I, 180 \mu \mathrm{m} ; \boldsymbol{B}, \boldsymbol{F}, J, N, 65 \mu \mathrm{m} ; C, G, K, 0,300 \mu \mathrm{m} ; \boldsymbol{D}, \boldsymbol{H}, \mathbf{L}, \mathbf{P}, 70 \mu \mathrm{m}$.

Azizi SA, Woodward DJ (1987) Inferior olivary nuclear complex of the rat: morphology and comments on the principles of organization within the olivocerebellar system. J Comp Neurol 263:467-484.

Backer S, Hidalgo-Sanchez M, Offner N, Portales-Casamar E, Debant A, Fort P, Gauthier-Rouviere C, Bloch-Gallego E (2007) Trio controls the mature organization of neuronal clusters in the hindbrain. J Neurosci 27:10323-10332.

Bloch-Gallego E, Ezan F, Tessier-Lavigne M, Sotelo C (1999) Floor plate and netrin-1 are involved in the migration and survival of inferior olivary neurons. J Neurosci 19:4407-4420.

Bourrat F, Sotelo C (1988) Migratory pathways and neuritic differentiation of inferior olivary neurons in the rat embryo. Axonal tracing study using the in vitro slab technique. Brain Res 467:19-37.

Bourrat F, Sotelo C (1990a) Migratory pathways and selective aggregation of the lateral reticular neurons in the rat embryo: a horseradish peroxidase in vitro study, with special reference to migration patterns of the precerebellar nuclei. J Comp Neurol 294:1-13.
Bourrat F, Sotelo C (1990b) Early development of the rat precerebellar system: migratory routes, selective aggregation and neuritic differentiation of the inferior olive and lateral reticular nucleus neurons. An overview. Arch Ital Biol 128:151-170.

Bourrat F, Sotelo C (1991) Relationships between neuronal birthdates and cytoarchitecture in the rat inferior olivary complex. J Comp Neurol 313:509-521.

Brose K, Bland KS, Wang KH, Arnott D, Henzel W, Goodman CS, TessierLavigne M, Kidd T (1999) Slit proteins bind Robo receptors and have an evolutionarily conserved role in repulsive axon guidance. Cell 96:795-806.

Camurri L, Mambetisaeva E, Davies D, Parnavelas J, Sundaresan V, Andrews W (2005) Evidence for the existence of two Robo3 isoforms with divergent biochemical properties. Mol Cell Neurosci 30:485-493.

Causeret F, Danne F, Ezan F, Sotelo C, Bloch-Gallego E (2002) Slit antagonizes netrin-1 attractive effects during the migration of inferior olivary neurons. Dev Biol 246:429-440. 

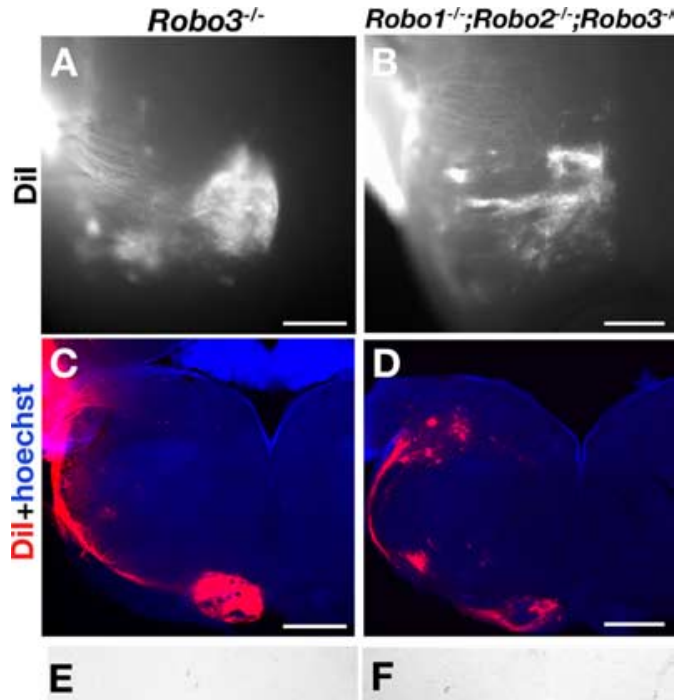

$\mathbf{F}$
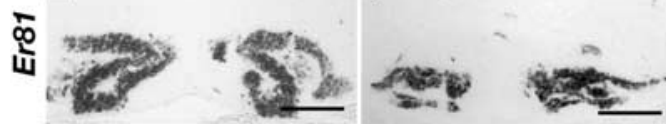

Figure 9. 10 projection and lamellation in Robo1/2/3 triple mutants. $A-D$, Ventral views of whole-mount brains $(\boldsymbol{A}, \boldsymbol{B})$ and coronal sections $(\boldsymbol{C}, \boldsymbol{D})$ from Dil-labeled E18 Robo $^{-/-}$single mutants $(\boldsymbol{A}, \boldsymbol{C})$ and Robo1/2/3 triple mutants $(\boldsymbol{B}, \boldsymbol{D})$. In both, the 10 nucleus is labeled on the ipsilateral side and 10 axons do not cross the midline. However, in the Robo $1 / 2 / 3$ triple mutants, the $I 0$ is less compact and fragmented. $\boldsymbol{E}, \boldsymbol{F}$, Coronal sections at the level of the $I 0$ of E18 Robo3 ${ }^{-1-}$ single mutant $(\boldsymbol{E})$ and Robo1/2/3 triple mutant $(\boldsymbol{F})$ hybridized with Er81 riboprobes. The 10 lamellation is still recognizable in Rob03 single mutant but not in Robo1/2/3 triple mutants. Scale bars: $A, B, 490 \mu \mathrm{m} ; \boldsymbol{C}, \boldsymbol{D}, 445 \mu \mathrm{m} ; \boldsymbol{E}, \boldsymbol{F}, 125 \mu \mathrm{m}$.
Challa AK, McWhorter ML, Wang C, Seeger MA, Beattie CE (2005) Robo3 isoforms have distinct roles during zebrafish development. Mech Dev 122:1073-1086.

Chedotal A, Pourquie O, Ezan F, San Clemente H, Sotelo C (1996) BEN as a presumptive target recognition molecule during the development of the olivocerebellar system. J Neurosci 16:3296-3310.

Chen Z, Gore BB, Long H, Ma L, Tessier-Lavigne M (2008) Alternative splicing of the Robo3 axon guidance receptor governs the midline switch from attraction to repulsion Neuron 58:325-332.

de Diego I, Kyriakopoulou K, Karagogeos D, Wassef M (2002) Multiple influences on the migration of precerebellar neurons in the caudal medulla. Development 129:297-306.

Dickson BJ (2002) Molecular mechanisms of axon guidance. Science 298:1959-1964.

Dickson BJ, Gilestro GF (2006) Regulation of commissural axon pathfinding by slit and its Robo receptors. Annu Rev Cell Dev Biol 22:651-675.

Ellenberger Jr C, Hanaway J, Netsky MG (1969) Embryogenesis of the inferior olivary nucleus in the rat: a radioautographic study and a reevaluation of the rhombic lip. J Comp Neurol 137:71-79.

Engelkamp D, Rashbass P, Seawright A, van Heyningen V (1999) Role of Pax6 in development of the cerebellar system. Development 126:3585-3596.

Engle EC (2007) Oculomotility disorders arising from disruptions in brainstem motor neuron development. Arch Neurol 64:633-637.

Fouquet C, Di Meglio T, Ma L, Kawasaki T, Long H, Hirata T, Tessier-Lavigne M, Chedotal A, Nguyen-Ba-Charvet KT (2007) Robol and robo2 control the development of the lateral olfactory tract. J Neurosci 27:3037-3045.

Furrer MP, Kim S, Wolf B, Chiba A (2003) Robo and Frazzled/DCC mediate dendritic guidance at the CNS midline. Nat Neurosci 6:223-230.

George L, Chaverra M, Todd V, Lansford R, Lefcort F (2007) Nociceptive sensory neurons derive from contralaterally migrating, fate-restricted neural crest cells. Nat Neurosci 10:1287-1293.

Goodman CS (1994) The likeness of being: phylogenetically conserved molecular mechanisms of growth cone guidance. Cell 78:353-356.

Grieshammer U, Le M, Plump AS, Wang F, TessierLavigne M, Martin GR (2004) SLIT2-mediated ROBO2 signaling restricts kidney induction to a single site. Dev Cell 6:709-717.

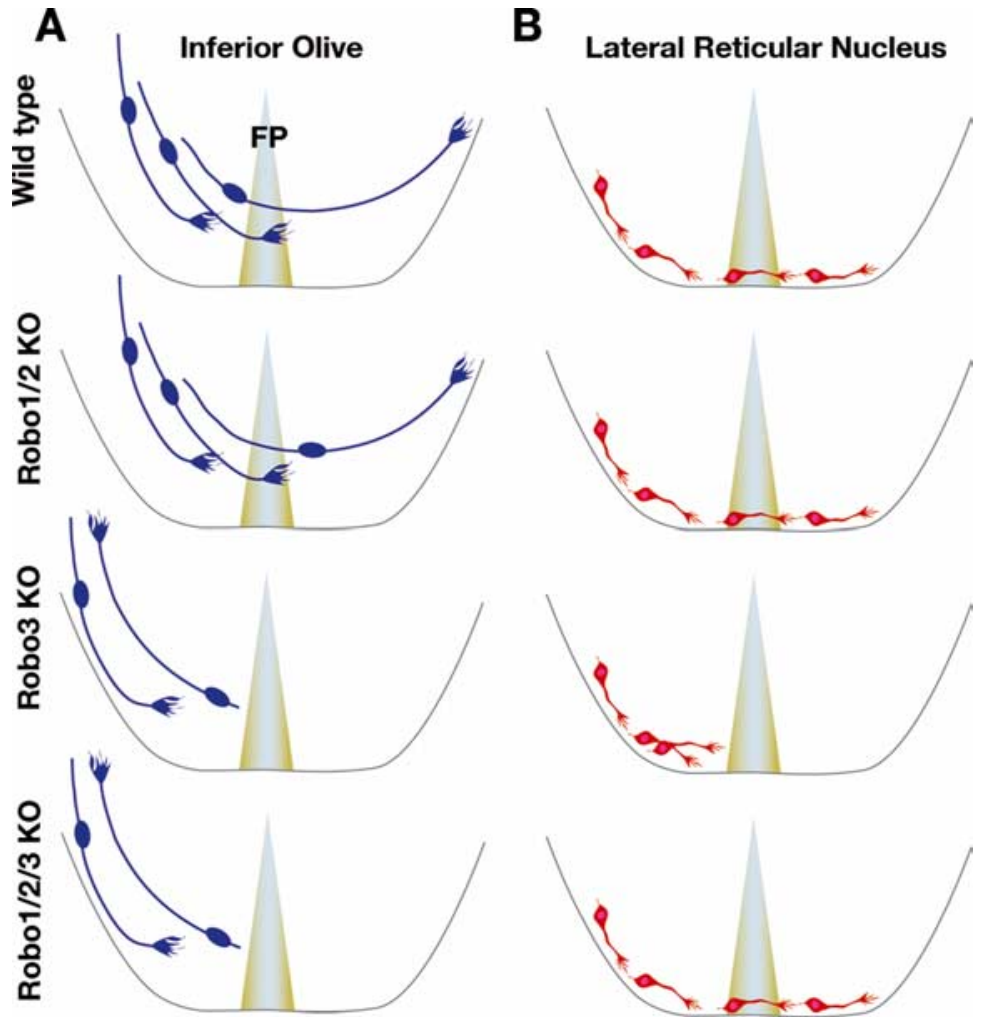

Figure 10. Schematic representation of 10 and LRN development in Robo mutants. $A$, In wild type, the axon of $I 0$ neurons cross the floor plate but their cell body does not. In Robo $1 / 2$ double mutants, all I 0 axons and a subset of 10 neurons cross the floor plate because of a reduction of midline repulsion. In contrast, in Robo3 single mutant and in Robo 1/2/3 triple mutant, 10 axons and neurons are unable to cross the midline. 10 axons project to the ipsilateral cerebellum. $\boldsymbol{B}$, In wild type and Robo1/Robo2 double mutants, all LRN axons and neurons cross the floor plate. In Robo3 single mutant, LRN axons and neurons stay ipsilateral and do not cross the floor plate. In Robo1/2/3 triple mutants, midline crossing is restored for at least some LRN axons and neurons.
Harkmark W (1954) Cell migrations from the rhombic lip to the inferior olive, the nucleus raphe and the pons; a morphological and experimental investigation on chick embryos. J Comp Neurol 100:115-209.

His W (1890) Die entwicklung des menschlichen Rautenhirns vom ende des ersten bis zum beginn des dritten monats. I. Verlangertes Mark. Abh Kg Sächs Ges Wissensch Math Phys Kl 29:1-74.

Hivert B, Liu Z, Chuang CY, Doherty P, Sundaresan V (2002) Robo1 and Robo2 are homophilic binding molecules that promote axonal growth. Mol Cell Neurosci 21:534-545.

Jen JC, Chan WM, Bosley TM, Wan J, Carr JR, Rub U, Shattuck D, Salamon G, Kudo LC, Ou J, Lin DD, Salih MA, Kansu T, Al Dhalaan H, Al Zayed Z, MacDonald DB, Stigsby B, Plaitakis A, Dretakis EK, Gottlob I, et al. (2004) Mutations in a human ROBO gene disrupt hindbrain axon pathway crossing and morphogenesis. Science 304:1509-1513.

Kawauchi D, Taniguchi H, Watanabe H, Saito T, Murakami F (2006) Direct visualization of nucleogenesis by precerebellar neurons: involvement of ventricle-directed, radial fibreassociated migration. Development 133:1113-1123.

Keleman K, Ribeiro C, Dickson BJ (2005) Comm function in commissural axon guidance: cellautonomous sorting of Robo in vivo. Nat Neurosci 8:156-163.

Kidd T, Brose K, Mitchell KJ, Fetter RD, Tessier- 
Lavigne M, Goodman CS, Tear G (1998) Roundabout controls axon crossing of the CNS midline and defines a novel subfamily of evolutionarily conserved guidance receptors. Cell 92:205-215.

Li S, Qiu F, Xu A, Price SM, Xiang M (2004) Barhll regulates migration and survival of cerebellar granule cells by controlling expression of the neurotrophin-3 gene. J Neurosci 24:3104-3114.

Lindwall C, Fothergill T, Richards LJ (2007) Commissure formation in the mammalian forebrain. Curr Opin Neurobiol 17:3-14.

Long H, Sabatier C, Ma L, Plump A, Yuan W, Ornitz DM, Tamada A, Murakami F, Goodman CS, Tessier-Lavigne M (2004) Conserved roles for Slit and Robo proteins in midline commissural axon guidance. Neuron 42:213-223.

Lopez-Bendito G, Flames N, Ma L, Fouquet C, Di Meglio T, Chedotal A, Tessier-Lavigne M, Marin O (2007) Robo1 and Robo2 cooperate to control the guidance of major axonal tracts in the mammalian forebrain. J Neurosci 27:3395-3407.

Lundstrom A, Gallio M, Englund C, Steneberg P, Hemphala J, Aspenstrom P, Keleman K, Falileeva L, Dickson BJ, Samakovlis C (2004) Vilse, a conserved Rac/Cdc42 GAP mediating Robo repulsion in tracheal cells and axons. Genes Dev 18:2161-2171.

Marillat V, Cases O, Nguyen-Ba-Charvet KT, Tessier-Lavigne M, Sotelo C, Chedotal A (2002) Spatiotemporal expression patterns of slit and robo genes in the rat brain. J Comp Neurol 442:130-155.

Marillat V, Sabatier C, Failli V, Matsunaga E, Sotelo C, Tessier-Lavigne M, Chedotal A (2004) The slit receptor Rig-1/Robo3 controls midline crossing by hindbrain precerebellar neurons and axons. Neuron 43:69-79.

McEvilly RJ, Erkman L, Luo L, Sawchenko PE, Ryan AF, Rosenfeld MG (1996) Requirement for Brn-3.0 in differentiation and survival of sensory and motor neurons. Nature 384:574-577.

Nishida K, Flanagan JG, Nakamoto M (2002) Domain-specific olivocerebellar projection regulated by the EphA-ephrin-A interaction. Development 129:5647-5658.

Orgogozo V, Schweisguth F, Bellaiche Y (2004) Slit-Robo signalling prevents sensory cells from crossing the midline in Drosophila. Mech Dev 121:427-436.

Paul LK, Brown WS, Adolphs R, Tyszka JM, Richards LJ, Mukherjee P, Sherr EH (2007) Agenesis of the corpus callosum: genetic, developmental and functional aspects of connectivity. Nat Rev Neurosci 8:287-299.

Plump AS, Erskine L, Sabatier C, Brose K, Epstein CJ, Goodman CS, Mason CA, Tessier-Lavigne M (2002) Slit1 and Slit2 cooperate to prevent pre- mature midline crossing of retinal axons in the mouse visual system. Neuron 33:219-232.

Rhee J, Mahfooz NS, Arregui C, Lilien J, Balsamo J, VanBerkum MF (2002) Activation of the repulsive receptor Roundabout inhibits $\mathrm{N}$-cadherinmediated cell adhesion. Nat Cell Biol 4:798-805.

Sabatier C, Plump AS, Le M, Brose K, Tamada A, Murakami F, Lee EY, Tessier-Lavigne M (2004) The divergent Robo family protein rig-1/ Robo3 is a negative regulator of slit responsiveness required for midline crossing by commissural axons. Cell 117:157-169.

Santiago-Martinez E, Soplop NH, Kramer SG (2006) Lateral positioning at the dorsal midline: Slit and Roundabout receptors guide Drosophila heart cell migration. Proc Natl Acad Sci USA 103:12441-12446.

Simon H, Lumsden A (1993) Rhombomere-specific origin of the contralateral vestibulo-acoustic efferent neurons and their migration across the embryonic midline. Neuron 11:209-220.

Suli A, Mortimer N, Shepherd I, Chien CB (2006) Netrin/DCC signaling controls contralateral dendrites of octavolateralis efferent neurons. J Neurosci 26:13328-13337.

Taniguchi H, Kawauchi D, Nishida K, Murakami F (2006) Classic cadherins regulate tangential migration of precerebellar neurons in the caudal hindbrain. Development 133:1923-1931.

Wassef M, Chedotal A, Cholley B, Thomasset M, Heizmann CW, Sotelo C (1992) Development of the olivocerebellar projection in the rat: I. Transient biochemical compartmentation of the inferior olive. J Comp Neurol 323:519-536

Watari-Goshima N, Ogura K, Wolf FW, Goshima Y, Garriga G (2007) C. elegans $\mathrm{VAB}-8$ and UNC-73 regulate the SAX-3 receptor to direct cell and growth-cone migrations. Nat Neurosci 10:169-176.

Williams SE, Mason CA, Herrera E (2004) The optic chiasm as a midline choice point. Curr Opin Neurobiol 14:51-60.

Wingate RJ (2001) The rhombic lip and early cerebellar development. Curr Opin Neurobiol 11:82-88.

Yee KT, Simon HH, Tessier-Lavigne M, O’Leary DM (1999) Extension of long leading processes and neuronal migration in the mammalian brain directed by the chemoattractant netrin-1. Neuron 24:607-622.

Yuan SS, Cox LA, Dasika GK, Lee EY (1999) Cloning and functional studies of a novel gene aberrantly expressed in RB-deficient embryos. Dev Biol 207:62-75.

Zhu Y, Guthrie S (2002) Expression of the ETS transcription factor ER81 in the developing chick and mouse hindbrain. Dev Dyn 225:365-368. 